\title{
DYNAMIC ANALYSIS OF INSULIN AND GLUCAGON SECRETIONS
}

\author{
Nan-Kuang Yao and Liang-Wey Chang* \\ Department of Electrical Engineering, College of Engineering \\ Center for Biomedical Engineering*, College of Medicine \\ National Taiwan University, Taipei 100, Taiwan.
}

\begin{abstract}
The interaction between insulin and glucagon secretions in an negative-positive relationship bolstered the concept that the net secretion of pancreatic hormones might be the product of specific interaction within the islet. However, how the interaction between $A$ and $B$ cells influences the secretion cycles is unclear. We developed a cell culture-microperifusion system that allowed us to study the detailed dynamics of plusatile secretions for both hormones. The power spectrum and cross-correlation analysis were used to investigate their oscillation features and phase relationship. The result showed that both insulin and glucagon secretions behaved in a spontaneous, persistent, regular, pulsatile and out-of-phase fashion. We suggest that insulin is dominant in the interactive process, i.e. , insulin is a master and glucagon is a slave.

Keywords: perifusion, insulin, glucagon, oscillation
\end{abstract}

\section{INTRODUCTION}

It is well known that the plasma glucose level is regulated by insulin and glucagon secretions. Glucagon stimulates glucose production, and insulin suppresses glucose production from the liver and increases glucose uptake in muscle and other tissues. Despite providing nearly opposite contributions to maintain fuel homeostasis, glucagon-secreting A cells (alpha) and insulin-secreting B cells (beta) are sophisticatedly aggregated in the islet structurally. More recently, careful three-dimensional reconstruction have shown that the human islet; like the rat islet, is composed of a core of B cells surrounded by a mantle of $A$ and $D$ cells. Because each of the major hormone affects the secretion of the other inside the islet [1], such a topography supports the hypothesis that the regulation of hormone secretion is based on intercellular communication. Furthermore, it was found that both the insulin and glucagon were secreted in an oscillatory fashion [2][3]. In this paper, we are to study if the phase relationship between insulin and glucagon exists and how the intercellular communication influences these secretion cycles. We developed a cell culture-microperifusion system to detect and quantify episodic hormone secretion [4]. This new system permitted us to investigate the detailed dynamic features of the pancreatic hormone secretions responded to various stimulators. In addition, the power spectrum and cross-correlation analysis were used to verify the relationship between insulin and glucagon.

\section{MATERIALS AND METHODS}

Intact islets were isolated from the pancreas of male Wistar rats (250-350 g) using the collagenase technique of Lacy and Kostianovsky with some modifications. Individual islets were hand-picked under the microscope and then cultured overnight in RPMI-1640 culture medium at $37^{\circ} \mathrm{C}, \mathrm{CO}_{2}$ incubators before being used. Details of the micro-perifusion procedure have been previously reported [4]. In brief, islets (80-100) were placed into a perifusion chamber made by $1 \mathrm{ml}$ plastic syringe. The bottom of the chamber consisted of 2 layers of $10 \mu \mathrm{m}$ nylon net and connected with a 21-gauge needle. Perifusion medium, the same as the medium used for overnight culture, was pumped through the chamber at a flow rate of 180 $\mu 1 / \mathrm{min}$. More than 100 Samples were collected at 3-min intervals. Insulin and glucagon concentrations were determined by the radioimmunoassay. Dextran-coated charcoal for insulin and second antibody for glucagon were used as separation methods.

\section{RESULTS}

When islets were continuously perifused with medium containing glucose changed from $100 \mathrm{mg} / \mathrm{dl}$ to $300 \mathrm{mg} / \mathrm{dl}$ in step function, insulin was suddenly increased and glucagon was decreased at the same time(data not shown). In order to analyze these oscillation phenomena further, islets were perifused with constant glucose concentration $(100 \mathrm{mg} / \mathrm{dl})$. Regular, consistent, cyclic rhythms of insulin and glucagon secretions were observed (Fig. 1). To reduce the noise due to assay error and, therefore, to render the main features more visible, the data were smoothed by cubic spline and fitted by 6-degree polynomial curves as detrending functions. Then the detrended data were divided by values predicted by detrending functions to produce the amplitude ratio curves(Fig. 2). Obviously, insulin and glucagon oscillations were changing in a similar trend both in amplitude ratio and period. This implied that the influence from interaction between insulin and glucagon really existed in their secretion cycles. The data were further processed by power spectrum analysis (Fig. 3) and autocorrelation (Fig. 4A,B). As a results, the regularity of the insulin and glucagon oscillations were confirmed and the period of the cycle for both hormones were almost the same. Mean period was $30 \pm$ $1.5 \mathrm{~min}$. The method of cross-correlation was used to 
determine phase shifts (Fig. 4C). The maximum correlation occurred $14.5 \mathrm{~min}$. later, which implied an out-of-phase relationship.

\section{DISCUSSION AND CONCLUSION}

The discovery that both insulin and glucagon affected the secretion of the other in a negative-positive relationship (insulin inhibit glucagon secretion, glucagon stimulate insulin secretion) bolstered the concept that the net secretion of pancreatic hormones might be the product of specific interaction within the islet. If one of the two hormones was dominant, which means its physiological effect might be faster or stronger than another one, the result would show different type of phase relationship. In the case of dominant insulin, the inhibition to A cells was faster and stronger. Glucagon secretion would immediately respond to the variation of insulin. Therefore, increased insulin would accompany with decreased glucagon and decreased change rate of insulin. Consequently, an out-of-phase oscillation fashion would occur. By the same reason, in the case of dominant glucagon, the stimulation to B cells would be faster and stronger. Insulin secretion immediately responded to the variation of Glucagon. This would bring about similar variation tendencies of both hormones, i.e., an inphase oscillations. Obviously, the data suggest that insulin is dominant in the interaction due to their out-of-phase oscillations. In a sense of "master and slave" relationship, insulin is a master and glucagon is a slave!

\section{ACKNOWLEDGMENTS}

This work was supported in part by the National Science Council under grant No. NSC 84 -2331-B-002-155 M08 and National Institute of Health under grant No. DOH 83-HR-310.

\section{REFERENCES}

[1] E. Samols, S. Bonner-Weir, G. C. Weir, "Intra-islet Insulin-Glucagon-Somatostatin relationships", Clinics in Endocrinology and Metabolism, vol.15, no.1, pp. 33-58, 1986.

[2] H. F. Chou, E. Ipp, "Pulsatile Insulin Secretion in Isolated Rat Islets", Diabetes, vol. 39, pp. 112-117, 1990.

[3] R. W. Bergstrom, W. Y. Fujimoto, D. C. Teller, C. D. Haen, "Oscillation Insulin Secretion In Perifused Isolated Rat Islets." Am. J. Physiol., vol. 257, pp. E479-E485, 1989.

[4] N. K. Yao, L. W. Chang, "Detailed Dynamic Analysis of Pulsatile Secretion in Isolated Rat Islets by a Cell Culture-Microperifusion System", Annual Symposium of the Biomedical Engineering Society, Taiwan, R.O.C., pp. 195-196, 1994.

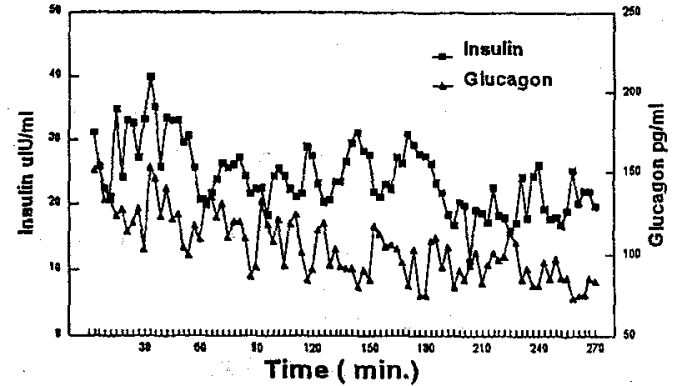

Fig. 1 Rhythm of insulin and glucagon secretions from isolated islets perifused with RPMI-1640 containing constant glucose, $100 \mathrm{mg} / \mathrm{dl}$.

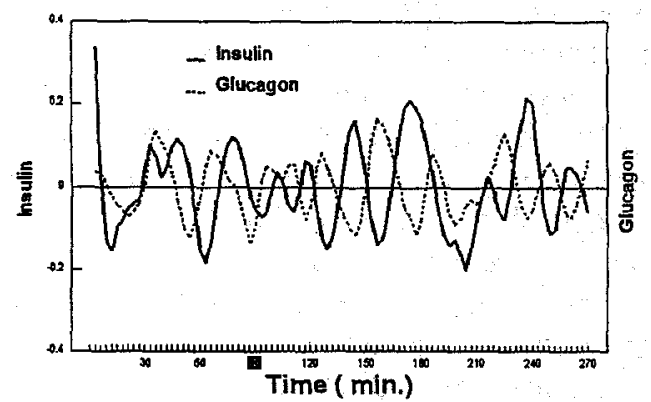

Fig. 2 Amplitude ratio curves of insulin and glucagon.
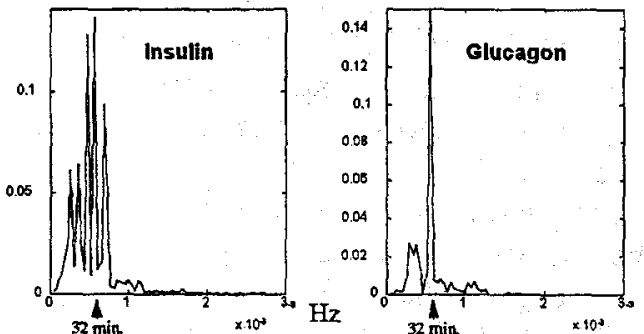

Fig. 3 Results of spectrum analysis of insulin and glucagon oscillations.
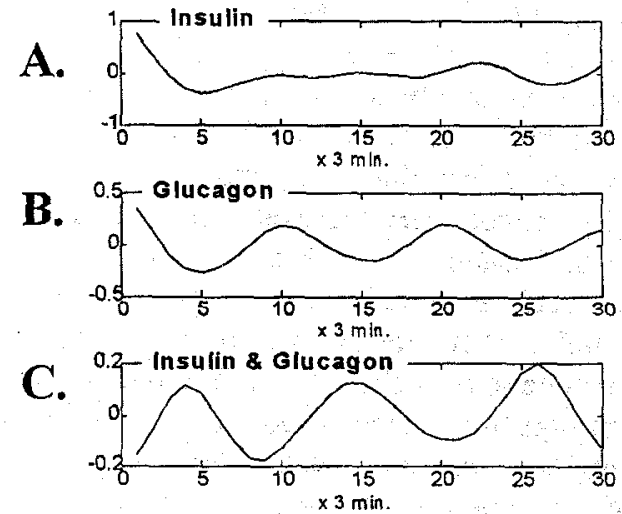

Fig. 4 Results of correlation analysis: (A) autocorrelation of insulin, (B) autocorrelation of glucagon, (C) crosscorrelation between insulin and glucagon. 